\title{
Diploid and triploid African catfish (Clarias gariepinus) differ in biomarker responses to the pesticide chlorpyrifos
}

\begin{abstract}
The impacts of environmental stressors on polyploid organisms are largely unknown. This study investigated changes in morphometric, molecular, and biochemical parameters in fullsibling diploid and triploid African catfish (Clarias gariepinus) in response to chlorpyrifos (CPF) exposures. Juvenile fish were exposed to three concentrations of CPF (mean measured $\mu \mathrm{g} / \mathrm{L}$ (SD): 9.71 (2.27), 15.7 (3.69), 31.21 (5.04)) under a static-renewal condition for 21 days. Diploid control groups had higher hepatosomatic index (HSI), plasma testosterone (T), and brain GnRH and cyp19a2 expression levels than triploids. In CPF-exposed groups, changes in HSI, total weight and length were different between the diploid and triploid fish. In contrast, condition factor did not alter in any of the treatments, while visceral-somatic index (VSI) changed only in diploids. In diploid fish, exposure to CPF did not change brain $11 \beta$-hsd2, ftz-f1, fox12, GnRH or cyp19a2 mRNA levels, while reduced tph2 transcript levels compared to the control group. In contrast, $11 \beta$-hsd 2 and foxl2 expression levels were changed in triploids following CPF exposures. In diploids, plasma $\mathrm{T}$ levels showed a linear dose-response reduction across $\mathrm{CPF}$ treatments correlating with liver weight and plasma total cholesterol concentrations. In contrast, no changes in plasma cholesterol and T concentrations were observed in triploids. Plasma cortisol and 17- $\beta$ estradiol (E2) showed no response to $\mathrm{CPF}$ exposure in either ploidy. Results of this first comparison of biomarker responses to pesticide exposure in diploid and polyploid animals showed substantial differences between diploid and triploid C. gariepinus.
\end{abstract}

Keyword: Biochemical; Gene expressions; Hormonal; Morphometric; Polyploidy 